\title{
The Pro-apoptotic Effects of S100A8 and S100A9 in Human Monocytic Leukemia Cells, THP-1
}

\author{
In-Sik Kim ${ }^{1,2}$ and Ji-Sook Lee $\mathrm{et}^{3, \dagger}$ \\ ${ }^{1}$ Department of Senior Healthcare, BK21 Plus Program, Graduate School, Eulji University, Daejeon 34824, Korea \\ ${ }^{2}$ Department of Biomedical Laboratory Science, School of Medicine, Eulji University, Daejeon 34824, Korea \\ ${ }^{3}$ Department of Clinical Laboratory Science, Wonkwang Health Science University, Iksan 54538, Korea
}

S100A8 and S100A9 are involved in pathogenesis of cancer by induction or inhibition of cancer as well as inflammation. In this study, we investigated the association of S100A8 and S100A9 with pathogenesis of leukemia using human monocytic leukemia cells, THP-1. The expression of TLR4, which is a known receptor of S100A8 and S100A9, was examined by using flow cytometry and Western blotting. THP-1 cells have high surface and cytosol expression of TLR4. S100A8 and S100A9 suppressed the cell survival, and this suppression was found to be associated with apoptosis because they increased the number of apoptotic cells in a dose- and a time-dependent manners. However, S100A8 and S100A9 had no effect on the survival and apoptosis of monocytes isolated from the peripheral blood. We next examined the apoptotic effect of lipopolysaccharide (LPS) and monophosphoryl lipid A (MPLA), which are other ligands of TLR4, in THP-1 cells. Lipopolysaccharide had no effect on cell survival, but MPLA is effective on the cell apoptosis. These results suggest that S100A8 and S100A9 may regulate leukemia cell survival via TLR4, which is an essential receptor in the pro-apoptotic mechanism induced by S100A8 and S100A9. These findings may shed light on development of a possible therapeutic drug for leukemia treatment.

Key Words: S100, Apoptosis, Leukemia

S100A8 and S100A9 are included in the S100 family of proteins and constitutively expressed in monocytes and neutrophils (Nam et al., 2016; Kim et al., 2017; Kim and Lee, 2017). Most studies of S100A8 and S100A9 have focused on their roles in inflammatory responses (Goyette and Geczy, 2011; Austermann et al., 2017). It has recently been demonstrated that S100A8 and S100A9 are closely related to the pathogenesis of cancer (Mao et al., 2014; Moris et al., 2016). For example, S100A8 and S100A9 increased in breast cancer, prostate cancer, and lung cancer (Leanderson et al., 2015). Moreover, S100A8 and S100A9 induce metastasis of cancer cells and inhibition of their function suppresses migration and invasion of cancer cells (Lim et al., 2016). Because S100A8 and S100A9 mediate their mechanism via Toll-like receptor 4 (TLR4), drug targeting based on inhibition of TLR4 has been conducted (Maru et al., 2015). However, other S100 family proteins such as S100A2 block squamous cell carcinoma through cyclooxygenase-2 (Tsai et al., 2006). In recent leukemia research, Laouedj et al. suggested that S100A9 induces differentiation of acute myeloid leukemia cells through TLR4 (Laouedj et al., 2017). Although the effects of S100A8 and S100A9 on monocytic leukemia are

* Received: May 17, 2018 / Accepted: May 28, 2018

${ }^{\dagger}$ Corresponding author: Ji-Sook Lee. Department of Clinical Laboratory Science, Wonkwang Health Science University, 501, Iksandaero, Iksan 54538, Korea. Tel: +82-63-840-1216, Fax:+82-63-840-1219, e-mail: jslee1216@wu.ac.kr (C) The Korean Society for Biomedical Laboratory Sciences. All rights reserved.

(C) This is an Open Access article distributed under the terms of the Creative Commons Attribution Non-Commercial License (http://creativecommons.org/licenses/by-nc/3.0/) which permits unrestricted non-commercial use, distribution, and reproduction in any medium, provided the original work is properly cited. 
(A)

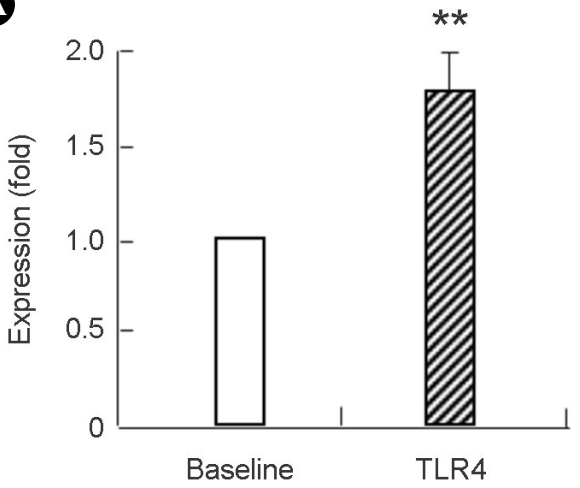

important in the pathogenesis or drug development of this disease, they have remained to be unveiled. Therefore, we examined the effects of S100A8 and S100A9 on apoptosis of human monocytic leukemia cells, THP-1, derived from an acute monocytic leukemia patient.

For detection of TLR4 expression, we conducted flow cytometry and Western blotting. In flow cytometry, the cells were treated with S100A8 or S100A9, collected and then fixed with $100 \mu \mathrm{L}$ of $0.37 \%$ paraformaldehyde solution for $15 \mathrm{~min}$ at room temperature. Following removal of the fixing solution, cells were washed twice with PBS buffer containing $0.5 \%$ BSA. The cells were subsequently separated into new tubes, to which PBS buffer containing anti-TLR4 antibodies (Santa Cruz Biotechnology, Santa Cruz, CA, USA) was added. Baseline fluorescence was obtained by incubation with normal mouse IgG instead of anti-TLR4 antibodies. Following three washes, the cells were incubated at $4{ }^{\circ} \mathrm{C}$ for $30 \mathrm{~min}$ with FITC-conjugated goat anti-mouse IgG (Molecular Probes; Eugene, OR, USA). Finally, the cells were washed and analyzed on a FACSort cytofluorimeter (Becton Dickinson). For Western blotting, the cells were treated with S100A8 or S100A9, harvested and lysed in an extraction buffer. The protein samples were separated by SDS-polyacrylamide gel electrophoresis, after which the transferred membranes were incubated with anti-TLR4 antibodies and then developed using the enhanced chemiluminescence detection system (Amersham Pharmacia Biotech). The same blot was stripped and reprobed with anti- $\beta$-actin antibodies (Santa Cruz Biotechnology) for use as an internal control. Survival rate was measured by MTT assay (Sigma
THP-1

Fig. 1. THP-1 cells express TLR4. (A) The expression of TLR4 was detected in THP-1 cells using flow cytometry (A) and Western blotting (B). Data are expressed as the means $\pm \mathrm{SD}$ and are presented relative to the baseline, which was set at $100 \%$. $* * P<0.01$ indicates a significant difference between the baseline and TLR4 expression (A).
Korea, Seoul, Korea). An annexin V-fluorescein isothiocyanate (FITC) apoptosis detection kit (BD Biosciences, San Diego, CA, USA) was used to detect the apoptosis of THP-1 cells and monocytes. Briefly, cells were incubated with FITC-labeled annexin V and propidium iodide (PI) for 15 min at room temperature, after which apoptotic cells were analyzed using a FACSCalibur with CellQuest software (BD bioscience) and then determined as the percentage of cells showing annexin $\mathrm{V}+/ \mathrm{PI}-$ and annexin $\mathrm{V}+/ \mathrm{PI}+$. A monocyte isolation kit II (Miltenyi Biotec) was used for isolation of monocytes from peripheral mononuclear cells. This study was approved by the Institutional Review Board of Eulji University for normal volunteers. All participants in this study gave their written informed consent. Values are expressed as the means \pm standard deviation (SD). Intergroup differences were analyzed by applying the Student's $t$-test within the SPSS software, version 18.0 (SPSS, Chicago, IL, USA). A $P<0.05$ was considered to indicate statistical significance.

We first investigated whether TLR4 exists in THP-1 cells. As shown in Fig. 1, TLR4 was expressed on the surface of THP-1 cells as well as within the cells. S100A8 or S100A9 decreased the survival rate of THP-1 cells in a time- and dose-dependent manners (Fig. 2A). To confirm the association of decreased survival with apoptosis, we evaluated the apoptotic rate of THP-1 cells after treatment with S100A8 or S100A9. Both proteins increased cell apoptosis in timeand dose-dependent manners after single and combined treatment (Fig. 2B and C). We previously reported that S100A8 and S100A9 increase the secretion of cytokines such as IL-6 IL-8, and MCP-1, which suppresses neutrophil apoptosis 


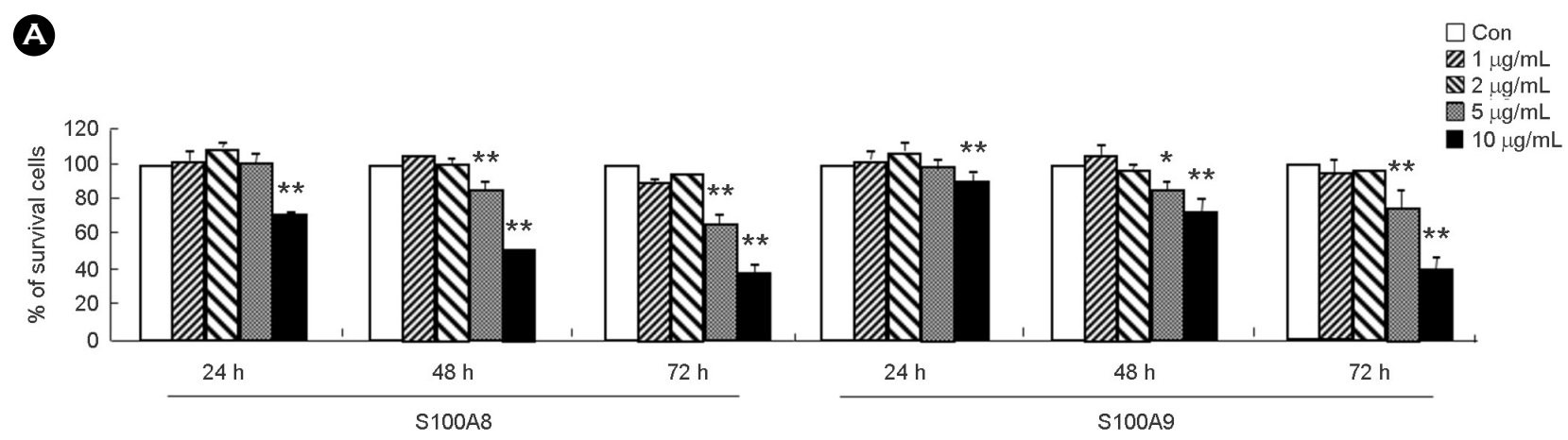

B

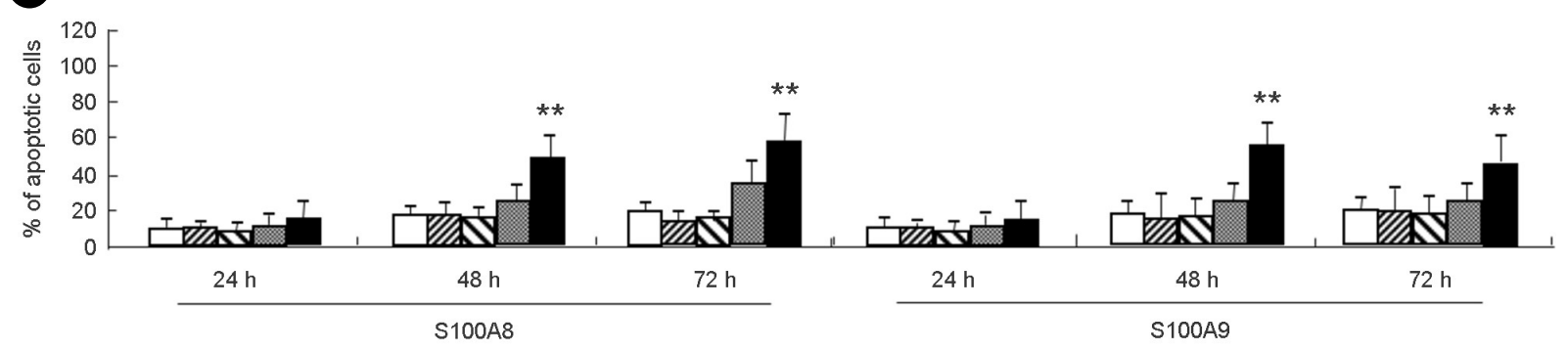

C

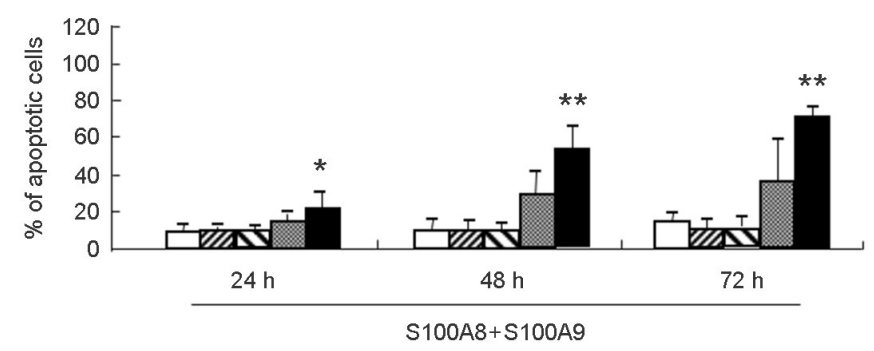

(D)

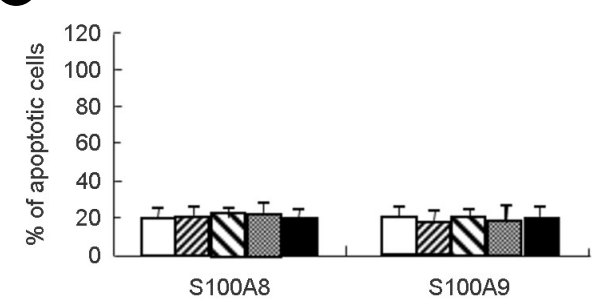

Fig. 2. Pro-apoptotic effects of S100A8 and S100A9 in THP-1 cells and monocytes. THP-1 cells (A-C) and monocytes (D) were incubated for the indicated time in the absence (Con) and presence of S100A8 or/and S100A9 at the indicated concentration. The survival rate (A) and apoptotic rate (B-D) were measured by MTT assay and apoptosis detection kit, respectively. Data are presented relative to the control, which was set at $100 \%$ (A). Data are expressed as the means \pm SD. $* P<0.05$ and $* * P<0.01$ indicate a significant difference between the control and stimulator-treated groups (A-D).

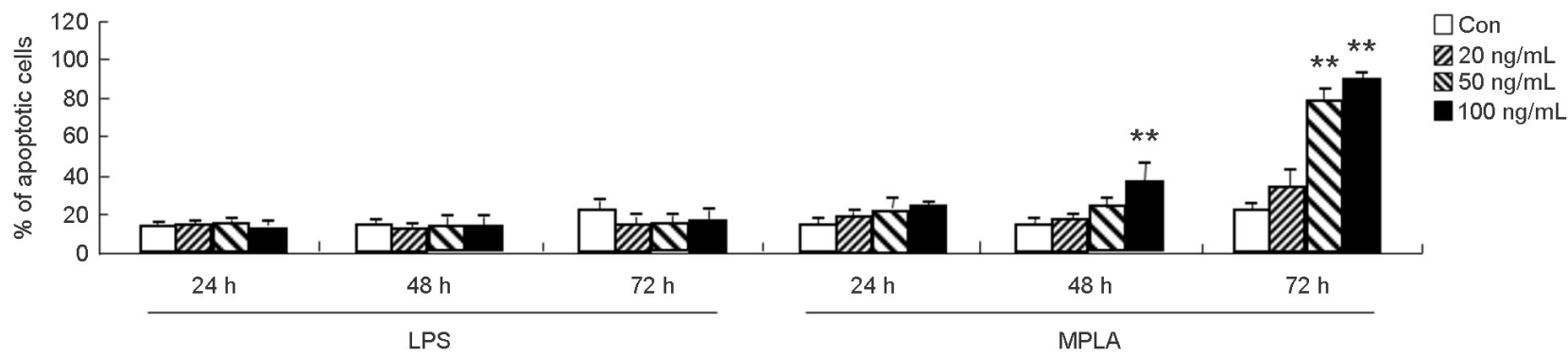

Fig. 3. Pro-apoptotic effect of LPS and MPLA in THP-1 cells. THP-1 cells were incubated for the indicated times in the absence (Con) and presence of LPS or MPLA at the indicated concentrations. Apoptotic rate was measured using an apoptosis detection kit. Data are expressed as the means $\pm \mathrm{SD}$, and $* P<0.05$ and $* * P<0.01$ indicate a significant difference between the control and stimulator-treated groups. 
(Lee et al., 2016). In the present study, we found that cytokine concentration strongly increases at $6 \mathrm{~h}$ after treatment with S100A8 and S100A9, then continues to diminish until $48 \mathrm{~h}$. Taken together, these findings indicate that S100A8 and S100A9 may induce inflammatory responses at an early stage and trigger cell apoptosis at a late stage. Apoptosis of normal monocytes from the peripheral blood is not altered by S100A8 and S100A9 (Fig. 2D). Therefore, we examined whether other TLR4 agonists affect apoptosis of THP-1 cells. Monophosphoryl lipid A (MPLA) is also effective against apoptosis; however, LPS did not show any apoptotic effect (Fig. 3). A recent study demonstrated that LPS and MPLA differentially regulate the functions of cytotoxic $\mathrm{T}$ cells (Weiguo et al., 2014). However, further study is needed to unveil whether TLR4-mediated mechanisms involving S100A8 and S100A9 are associated with Myd88-dependent or Myd88independent signal transduction.

\section{ACKNOWLEDGEMENT}

This paper was supported by Wonkwang Health Science University in 2018.

\section{CONFLICT OF INTEREST}

The authors have no conflicts of interest, financial or otherwise, to declare.

\section{REFERENCES}

Austermann J, Zenker S, Roth J. S100-alarmins: potential therapeutic targets for arthritis. Expert Opin Ther Targets. 2017. 21: 739-751.

Goyette J, Geczy CL. Inflammation-associated S100 proteins: new mechanisms that regulate function. Amino Acids. 2011. 41: 821-842.

Kim IS, Gu A, Lee JS. The role of S100A8 and S100A9 in differentiation of human eosinophilic leukemia cells, EoL-1. Biomed Sci Lett. 2017. 23: 44-47.

Kim IS, Lee JS. S100A8 and S100A9 secreted by allergens in monocytes inhibit spontaneous apoptosis of normal and asthmatic neutrophils via the Lyn/akt/ERK pathway. Korean J Clin Lab Sci. 2017. 49: 128-134.

Laouedj M, Tardif MR, Gil L, Raquil MA, Lachhab A, Pelletier
M, Tessier PA, Barabé F. S100A9 induces differentiation of acute myeloid leukemia cells through TLR4. Blood. 2017. 129: 1980-1990.

Leanderson T, Liberg D, Ivars F. S100A9 as a Pharmacological Target Molecule in Inflammation and Cancer. Endocr Metab Immune Disord Drug Targets. 2015. 15: 97-104.

Lee NR, Park BS, Kim SY, Gu A, Kim DH, Lee JS, Kim IS. Cytokine secreted by S100A9 via TLR4 in monocytes delays neutrophil apoptosis by inhibition of caspase $9 / 3$ pathway. Cytokine. 2016. 86: 53-63.

Lim SY, Yuzhalin AE, Gordon-Weeks AN, Muschel RJ. Tumorinfiltrating monocytes/macrophages promote tumor invasion and migration by upregulating S100A8 and S100A9 expression in cancer cells. Oncogene. 2016. 35: 5735-5745.

Mao Y, Poschke I, Kiessling R. Tumour-induced immune suppression: role of inflammatory mediators released by myelomonocytic cells. J Intern Med. 2014. 276: 154-170.

Maru Y, Tomita T, Deguchi A, Ieguchi K, Takita M, Tsukahara F, Takemura K, Kitao A, Gusovsky F. Drug Targeting Based on a New Concept-Targeting Against TLR4 as an Example. Endocr Metab Immune Disord Drug Targets. 2015. 15: 83-87.

Moris D, Spartalis E, Angelou A, Margonis GA, Papalambros A, Petrou A, Athanasiou A, Schizas D, Dimitroulis D, Felekouras E. The value of calprotectin S100A8/A9 complex as a biomarker in colorectal cancer: A systematic review. J BUON. 2016. 21: 859-866.

Nam AR, Kim DH, Kim MJ, Lee JS, Yang SJ, Kim IS. S100A8 induces secretion of MCP-1, IL-6, and IL-8 via TLR4 in Jurkat T cells. Biomed Sci Lett. 2016. 22: 60-64.

Tsai WC, Tsai ST, Jin YT, Wu LW. Cyclooxygenase-2 is involved in S100A2-mediated tumor suppression in squamous cell carcinoma. Mol Cancer Res. 2006. 4: 539-547.

Weiguo Cui, Nikhil S. Joshi, Ying Liu, Hailong Meng, Steven H Kleinstein, Susan M. Kaech. TLR4 ligands LPS and MPLA differentially regulate effector and memory CD8+ T cell differentiation J Immunol. 2014. 192: 4221-4232.

https://doi.org/10.15616/BSL.2018.24.2.134

Cite this article as: Kim IS, Lee JS. The Pro-apoptotic Effects of S100A8 and S100A9 in Human Monocytic Leukemia Cells, THP-1. Biomedical Science Letters. 2018. 24: 134-137. 